\title{
IDENTIFICAÇ̃̃O PRECOCE DOS FATORES DE RISCO PARA LESÕES POR PRESSÃO: PERCEPÇÃO DA EFETIVIDADE PELOS ENFERMEIROS
}

\section{EARLY IDENTIFICATION OF RISK FACTORS FOR PRESSURE INJURIES: PERCEPTION OF EFFECTIVENESS BY NURSES}

Adriana Anastácia dos Santos Dias ${ }^{1}$, Sheila Nara Ferreira ${ }^{2}$, Lilian Machado Torres $^{3^{*}}$

${ }^{1}$ Enfermeira. Pós-graduanda em Urgência e Emergência, ênfase em Cardiologia, pelo IEP - Santa Casa de Belo Horizonte.

2 Enfermeira. Especialista em Assistência de Enfermagem de média e alta complexidade - Estomaterapia pela Universidade Federal de Minas Gerais.

${ }^{3}$ Enfermeira. Pedagoga. Doutora em Ciências pela Escola de Enfermagem da USP. Especialista em Administração Hospitalar pelas Organizações São Camilo. Especialista em Metodologia do Ensino Superior pela PUC Minas. Especialista em Epidemiologia do Controle de Infecção Hospitalar pela PUC Minas.

* Alameda Antares, 480. Bairro Ville de Montagne. Nova Lima. Minas Gerais. CEP 34004-882. Email: lilian.torres0806@gmail.com

Submetido em: 17/10/2019; Aceito em:13/03/2020.

\section{RESUMO}

A prevenção de lesões por pressão é relevante para o cuidado holístico em saúde. A pronta identificação de fatores de risco que levam às lesões e o registro das observações, permite aos profissionais, em especial, aos enfermeiros, a adoção imediata de medidas preventivas. O objetivo do estudo foi analisar a compreensão do enfermeiro sobre a efetividade da identificação precoce dos fatores de risco de Lesão por Pressão por meio de instrumentos disponíveis. Trata-se de um estudo qualitativo, realizado em uma instituição hospitalar de grande porte na cidade de Belo Horizonte, MG, a partir de entrevistas guiadas por um roteiro. Participaram 13 enfermeiros que verbalizaram sobre seu cotidiano na prevenção de lesões. Do processo de análise do conteúdo expresso emergiram as seguintes categorias: Identificando e avaliando fatores de risco para as lesões; outras ferramentas utilizadas pela equipe de enfermagem; e $\mathrm{O}$ olhar mais amplo para além das dificuldades na prevenção de lesões por pressão. Conclui-se ser urgente tornar mais claro, desde a formação dos profissionais, o papel fundamental da prevenção de lesões a partir do conhecimento e identificação dos fatores de risco. É preciso aproximar o saber das ações necessárias para a qualificar o cuidado.

Palavras-chave: Enfermagem. Estomaterapia. Gestão da Segurança. Lesão por Pressão. Segurança do Paciente. 


\begin{abstract}
The prevention of pressure injuries is relevant to the holistic health care. The ready identification of risk factors that lead to injuries and the record of observations allow professionals, in particular, nurses, to adopt immediately preventive measures. The objective of this study was to analyze nurses' understanding about the effectiveness of early identification of risk factors of pressure injury through the available instruments. This is a qualitative study, performed at a large-sized hospital institution in the city of Belo Horizonte, MG, from interviews carried out by a guide. The participants were 13 nurses who verbalized about their everyday life in injury prevention. The process of analysis of the expressed contents originated the following categories: Identifying and evaluating risk factors for injuries; other tools used by the nursing staff; and the broader look beyond the difficulties in the prevention of pressure injuries. Therefore, there is an urgent need to make clearer the fundamental role of prevention of injuries from the knowledge and identification of risk factors since professionals' training. One must approach the knowledge to the actions necessary to qualify the care.
\end{abstract}

Keywords: Nursing. Stomatherapy. Safety Management. Pressure Ulcer. Patient Safety.

\title{
INTRODUÇÃO
}

Muito se discute sobre os aspectos que envolvem a qualidade dos cuidados em saúde e, em função disso, a Segurança do Paciente (SP) configura-se preocupação mundial (BRASIL, 2014). Dados apontam que em países desenvolvidos um em cada 10 indivíduos é prejudicado ao receber atendimento hospitalar. O risco de infecção associado aos cuidados de saúde, por exemplo, em alguns países em desenvolvimento, é de até 20 vezes maior que em países desenvolvidos (SILVA et al., 2016). Paralelamente, autores afirmam que a incidência de eventos adversos no Brasil é de $7,6 \%$, e que $66,7 \%$ deles poderiam ser evitados, como administração incorreta de medicamentos, falhas na identificação dos indivíduos, quedas, erros em procedimentos cirúrgicos, uso inadequado de equipamentos de saúde e úlceras por pressão (MASCARENHAS; MELO, 2015).

Estudo brasileiro que analisou 53 pesquisas conduzidas por enfermeiros sobre SP, disponíveis no Catálogo de Teses e Dissertações da Associação Brasileira de Enfermagem, detectou tendência de investigações em ambiente hospitalar, com ênfase na redução do risco de úlcera por pressão (GOMES et al., 2017). Tal evento adverso, cuja terminologia foi alterada para Lesão por Pressão (LP) (MORAES et al., 2016), refere-se a um dano causado na pele íntegra ou ulcerada ou em tecidos moles subjacentes. Geralmente ocorre sobre uma proeminência óssea, combinada com cisalhamento (dobras na pele quando o corpo desliza no leito) ou relacionada ao uso de instrumentos médicos ou outro objeto (VASCONCELOS; CALIRI, 2017).

No boletim brasileiro sobre Segurança do Paciente e Qualidade em Serviços de Saúde os eventos graves notificados (aqueles que nunca deveriam 
ocorrer em serviços de saúde), ou seja, os neverevents, totalizaram 216 e observa-se que a maioria das notificações a eles referentes $(94,8 \%)$ consistiuse em LP, nos estágios III e IV (BRASIL, 2017a).

A pronta identificação de indivíduos em risco e o registro das observações, permite à equipe de saúde a adoção imediata de medidas preventivas. E os registros, mais que exigências ético-legais, possibilitam a elaboração do plano de cuidados para o restabelecimento da saúde e sua avaliação, por meio do acompanhamento da evolução assistencial (MACHADO et al., 2017; VASCONCELOS; CALIRI, 2017).

Um dos instrumentos de avaliação mais utilizados pela equipe de Enfermagem, nas instituições hospitalares, é denominado Escala de Braden. Visa a identificação e pronta instalação de medidas de proteção para indivíduos em risco de desenvolvimento de lesões, a partir da Sistematização da Assistência de Enfermagem (SAE) (SALGADO et al., 2018).

Isso posto, surge uma questão: os enfermeiros que gerenciam e prestam o cuidado relacionam a identificação precoce de risco à implementação de medidas preventivas para a ocorrência de LP?

O estudo mostra-se relevante em função dos dados epidemiológicos e estatísticos que envolvem as referidas lesões, o que demonstra interesse dos profissionais sobre o tema, apesar do aumento de sua incidência. Analisar a compreensão do profissional sobre o cuidado sistematizado e que inclui o uso de instrumentos de identificação precoce de riscos, contribui para a segurança do indivíduo e para a redução da incidência do evento. Além disso, os resultados poderão subsidiar discussões na equipe de cuidadores e de gestores com vistas a um desenho de linha assistencial que considere cada indivíduo no processo saúde doença.

Isso posto, o objetivo do estudo foi compreender a percepção dos enfermeiros sobre a efetividade da identificação precoce dos fatores de risco de LP, a partir da utilização de instrumentos disponíveis em seu cotidiano.

\section{MATERIAL E MÉTODOS}

Estudo qualitativo que permite desvelar as questões no próprio ambiente em que elas se apresentam, sem qualquer manipulação intencional do pesquisador, retratando o maior número possível de elementos existentes na realidade estudada (PRODANOV; FREITAS, 2013).

A pesquisa foi realizada em um hospital na cidade de Belo Horizonte, Minas Gerais, pertencente a um complexo hospitalar. O referido serviço possui 156 leitos, Pronto Atendimento, Centro Cirúrgico e Centro de Tratamento Intensivo. São 19 enfermeiros que compõem a equipe assistencial aos adultos hospitalizados.

A coleta de dados ocorreu entre janeiro e fevereiro de 2018, após a aprovação nos Comitês de Ética em Pesquisa (CEP) da Faculdade Ciências Médicas de Minas Gerais e do Grupo Santa Casa de Belo Horizonte, sob os números 2.379.051 e 2.438.923, respectivamente.

Após a aprovação ética ocorreu a aproximação do pesquisador junto à unidade assistencial, quando a gerente recebeu todas as informações sobre a pesquisa e, então, disponibilizou o local, os melhores dias e horários e a escala 
dos profissionais para iniciar a coleta de dados.

Os participantes foram enfermeiros, maiores de 18 anos, que prestavam assistência direta aos indivíduos adultos hospitalizados. Foram excluídos os profissionais que estavam de férias ou licenças diversas durante o período de coleta de dados e aqueles que não atenderam a três tentativas para a realização da entrevista.

Posteriormente, após a concordância e assinatura dos participantes no Termo de Consentimento Livre Esclarecido, as entrevistas aconteceram em uma sala reservada, em que estiveram presentes somente o pesquisador e 0 participante, de modo a preservar sua privacidade. O tempo médio de cada encontro foi de 20 minutos. Houve gravação de toda a conversa, com autorização expressa dos depoentes.

Após a finalização dos depoimentos ocorreu a transcrição literal das entrevistas e a análise de conteúdo, no referencial de Laurence Bardin (BARDIN, 2018). Trata-se de um conjunto de técnicas de análise das comunicações, a partir de procedimentos sistemáticos, que levam à inferência de conhecimentos relativos às condições de produção/recepção (variáveis inferidas) das mensagens (CAMARA, 2013).

Os pesquisadores se comprometeram junto ao cenário de estudo a participar de ações específicas em função dos resultados obtidos. Considerase que a translação do conhecimento é parte integrante de estudos de campo. Os benefícios decorrentes do estudo envolvem a qualificação da assistência prestada, ao gerar melhorias no cuidado ao indivíduo em risco de desenvolver LP. Além disso, os gestores poderão usar os resultados para os programas de educação permanente.

\section{RESULTADOS E DISCUSSÃO}

Participaram 13 enfermeiros, a maioria mulheres e, apenas dois homens, com a média de idade de 32 anos. Do total, cinco profissionais com tempo de trabalho inferior a cinco anos e, oito deles, com tempo de profissão entre cinco e 10 anos.

Do processo de análise do conteúdo expresso emergiram as seguintes categorias: Identificando e avaliando fatores de risco para as lesões; outras ferramentas utilizadas pela equipe de Enfermagem; e $\mathrm{O}$ olhar mais amplo para além das dificuldades na prevenção de lesões por pressão.

\section{Identificando e avaliando fatores de risco para as lesões}

Os depoentes ressaltaram, mesmo que de forma incompleta, a existência de fatores de ordem intrínseca e extrínseca que contribuem para o desenvolvimento de LP. Fatores gerais, mais amplos e conhecidos, inerentes ao próprio indivíduo foram citados: "Existem fatores do próprio paciente: se está acamado, se já possui úlcera por pressão ou comorbidades, desidratação, desnutrição. Às vezes é um paciente sequelado, que ficou mais dependente nas atividades diárias" (ENF 2), "Indivíduo acamado, desnutrição, sobrepeso, alimentação inadequada, quadro de diarreia, eliminação urinária no leito. São todos fatores que considero predisposição para o aparecimento de lesão por pressão" (ENF 4). 
Outro participante, além de citar fatores intrínsecos, mencionou os extrínsecos que poderiam favorecer o surgimento de tais lesões:

"Os aspectos que favorecem o surgimento de lesão por pressão são a umidade, o uso de drogas vasoativas, as comorbidades, como hipertensão arterial e diabetes. São fatores intrínsecos e extrínsecos: o meio também, a exposição aos fluidos (referindo-se aos fluídos corpóreos)" (ENF 6).

A literatura estratifica as causas ligadas ao indivíduo e a fatores externos, além da interação deles, o que aumentaria a probabilidade do surgimento da lesão. Foi o que demonstrou um estudo realizado com 57 indivíduos internados em unidade de terapia intensiva cirúrgica que desenvolveram LP, durante 0 período de hospitalização. Os fatores predisponentes intrínsecos e extrínsecos transitaram entre imobilidade, choque séptico, uso de vasopressores, elevação da cabeceira a 30을 durante longo período de tempo, sedação e ventilação mecânica por mais de 72 horas, além da idade que pode contribuir significativamente (COX; ROCHE; MURPHY, 2018).

Os depoentes apontaram a efetividade da avaliação de risco para LP, quando afirmaram utilizar a escala de Braden, logo após a admissão hospitalar:

"Após a admissão faz-se a evolução de Enfermagem, nela existe a escala de Braden, utilizada para avaliar a predição do risco de desenvolver lesão por pressão, instrumento que permite um direcionamento. A conduta e o plano de cuidados são possíveis a partir daí. Pelo exame físico verifico quais são as demandas relacionadas à possibilidade de lesões para colocar as ações preventivas em prática" (ENF 3). "Temos um protocolo instituído: sempre na admissão é feita a avaliação $e$ temos o AGEIS: um sistema eletrônico da instituição que possui a escala de Braden. Então, é feita a avaliação de risco, além da nossa percepção como profissional, pois, o estado clínico vai influenciar muito na aplicabilidade dessa escala" (ENF 6).

Neste sentido, há uma multiplicidade de elementos que podem desencadear as lesões e dependem da utilização de instrumentos adequados para se obter uma avaliação eficaz dos fatores de risco como, por exemplo, a citada escala de Braden. Sua utilização é considerada pelos enfermeiros como um instrumento indispensável de avaliação, ao propiciar a elaboração de um plano de cuidados eficiente, além de ampliar a compreensão do cuidado associado às boas práticas. Outro depoente afirma:

"Na admissão de todos os pacientes é realizada a avaliação de risco pela escala de Braden, dentro do 
programa que se chama AGEIS. Ela é realizada de acordo com a anamnese, se ele está consciente, orientado.... mas quando não mantém contato, a nossa conversa é com o acompanhante. Também se verifica a nutrição, se tem alguma sonda e, depois, o exame físico. Com as informações coletadas identifica-se o risco de se desenvolver uma lesão por pressão. E o programa já fornece os resultados e os cuidados que devem ser realizados" (ENF 12).

De fato, pesquisas ressaltam que a escala de Braden contribui, de forma impar, para a prática segura, por associar-se à prevenção, que reduz a incidência de LP, ser de fácil manuseio, além de possuir baixo custo institucional, o que a torna um indicador de qualidade na assistência de Enfermagem (HOMMEL; SANTY-TOMLINSON, 2018; SALGADO et al., 2018; SOARES; HEIMANN, 2018).

Um dos enfermeiros, além de explicitar o uso da escala de Braden, ressaltou a questão do cuidado individualizado:

"Todos tem os cuidados direcionados pela Escala de Braden, pois o paciente é individual, não existe um cuidado geral para todos. É uma escala que tem uma efetividade muito significativa, uma vez que ela mesma me direciona para a individualidade. Essa avaliação deve ser feita a cada mudança dos fatores de risco, para nova classificação e direcionamento dos cuidados" (ENF 5).

Percebe-se a preocupação dos depoentes em relação à atenção individualizada ou assistência planejada para cada situação, em especial. Individualizar o cuidado relaciona-se a práticas seguras para prevenção de LP ao considerar que o cuidado emerge como eixo central da qualidade assistencial. O planejamento remete ao enfermeiro o atendimento qualificado, a partir de ações apropriadas para cada indivíduo, em conjunto com a família, tanto para prevenir, quanto para tratar lesões já instaladas, além de proporcionar uma linguagem homogênea na comunicação da equipe (AGYEMAN-YEBOAH; KORSAH; OKRAH, 2017; BRASIL, 2017b; SOARES; HEIMANN, 2018).

A aplicação de outras escalas para a avaliação de risco, também foi destacada pelos entrevistados:

"Existe também outra escala, a de Norton, mas de uma forma geral conseguimos ver a efetividade no decorrer do cuidado, ao aplicar essas escalas, pois ajuda bastante a cercar aqueles com mais risco. A reavaliação é diária, durante a corrida de leito aplicamos a escala para verificar se esse escore (referindo-se aos resultados aferidos nas escalas) melhora ou não. Fazemos um acompanhamento, com o olhar mais crítico e um maior cuidado" (ENF 1). 
"Tem no sistema da instituição Braden e Morse, que caminham juntas na hora de avaliar se existe alguma lesão. Também perguntamos e fazemos o exame físico. $O$ acompanhante ajuda quando o paciente não pode ou não sabe responder" (ENF 8).

De fato, existem várias outras escalas para a avaliação de risco de LP, como Norton, Waterlow, de Sunderland e Escala revista de Cubbin e Jackson. São consideradas ferramentas importantes e, a escolha de uma delas, depende do estado físico do indivíduo, patologia de base, unidade de trabalho, condições sociais, entre outros aspectos. Vale ressaltar que precisam ser sustentadas por profissionais capacitados e protocolos institucionais (ROCHA et al., 2016; SALGADO et al., 2018; ZIMMERMANN et al., 2018).

$\mathrm{Na}$ avaliação de risco o exame físico, a evolução e a prescrição de Enfermagem compõem o plano de cuidados. Nos depoimentos o exame físico apareceu em destaque: "Assim que se admite alguém é possível conhecer a história, fazer o exame físico, verificar o risco pela escala de Braden" (ENF 10), "Para todos os admitidos é realizado o exame físico, orientações para ele ou para o cuidador presente no momento. Avaliam-se as possíveis áreas de sofrimento, a Enfermagem evolui e utiliza a escala de Braden" (ENF 4).

Entre as dez práticas seguras para prevenção de LP está a avaliação de risco de todos os indivíduos antes e durante a internação, por meio do exame físico. Tal ação é capaz de identificar as necessidades atuais e futuras de cuidados, além de permitir o diagnóstico de problemas, a prescrição de cuidados de Enfermagem e a evolução. $O$ exame físico é considerado elemento básico para a prática clínica efetiva (BRASIL, 2017b; SALGADO et al., 2018).

Os indivíduos com maior risco para LP devem ser acompanhados diariamente e ter a pele avaliada logo na admissão e nos dias subsequentes, principalmente nos pontos de maior pressão corporal contra as superfícies. No entanto, a avaliação, por si só, não é suficiente para reduzir sua incidência, mas o monitoramento constante se antepõe aos problemas de integridade, promove a proteção da pele contra umidade, fricção e cisalhamento, além de direcionar a higienização e a hidratação (HOMMEL; SANTY-TOMLINSON, 2018).

A implementação das melhores práticas para a prevenção de LP tem sido associada à utilização de escalas de avaliação nos serviços de saúde, apesar dos desafios relacionados à sua utilização correta e contínua para que se torne, de fato, avaliação diária e precisa (VASCONCELOS; CALIRI, 2017). Não se pode deixar de ressaltar que proporcionam o planejamento de um cuidado individualizado e direcionado.

Paralelamente à exposição de condições inerentes ao indivíduo e relativas ao cuidado, emergiram ações que auxiliam na identificação de riscos. Os resultados também evidenciaram outras ferramentas indispensáveis para a organização do processo de Enfermagem e maior qualidade na assistência. 


\title{
Outras ferramentas utilizadas pela equipe de Enfermagem
}

No cenário pesquisado houve menção ao um comitê composto por profissionais de saúde e direcionado ao cuidado de lesões. Conforme o nível de classificação de risco do indivíduo, aciona-se o serviço de integridade cutânea, disponível na instituição:

\begin{abstract}
"Na corrida de leito observamos alterações do quadro clinico do paciente para que os cuidados estejam de acordo com a condição daquele momento. Quando identificamos o risco alto pedimos o acompanhamento do serviço de integridade cutânea" (ENF 9).
\end{abstract}

"Temos a comissão de integridade cutânea que acompanha os que precisam e realiza os curativos. Acompanha o desenvolvimento da lesão" (ENF 11), "Pacientes com o escore de risco para lesões são informadas ao serviço de integridade cutânea, uma equipe de enfermeiros que os acompanha diariamente. Indica-se a cobertura específica para o tipo de lesão" (ENF 12).

Os serviços especializados, ora denominados, em sua maioria, Comissão de Curativos, são considerados relevantes para prevenção e para o tratamento de LP. O enfermeiro especialista em lesões e coberturas, inserido na equipe multidisciplinar, que atua mediante protocolos baseados em evidências científicas, contribui para o aprimoramento dos demais profissionais (AGYEMAN-YEBOAH; KORSAH; OKRAH, 2017; MACHADO et al., 2017).

Além disso, os profissionais verbalizaram a importância de se agregar outras dimensões no acompanhamento do indivíduo hospitalizado, em conjunto com a comissão de integridade cutânea, que incluem a aplicação da SAE e os registros: "Utiliza-se SAE e comunica-se à comissão de integridade cutânea. Assim, os curativos acontecem segundo as orientações dessa comissão e do médico assistente" (ENF 10), "Reaplicamos a escala (referindo-se à escala de Braden) e acionamos, também, o serviço de integridade cutânea. Se estiver com o Braden de risco elevado, conseguimos interceptar bem antes de desenvolver a lesão" (ENF 7), "Faço os meus cuidados sistematizados de Enfermagem, aciono o serviço de integridade cutânea e converso com a família. É o que nos ajuda a prevenir a lesão" (ENF 2).

Um dos profissionais cita o registro como forma de acompanhamento:

"Tem a evolução (referindo-se aos registros de Enfermagem) que fazemos aqui, mas, a evolução não é diária. Verificarmos se as condições clínicas foram alteradas para que se adapte a escalação de profissionais e o plano de cuidados individual" (ENF 3).

Sistematizar a assistência, enquanto respaldo jurídico, considera que os registros constituem base de dados para novas práticas, competências e habilidades que transformam a realidade profissional. Torna-se fundamental no tratamento do indivíduo com LP a utilização da SAE, que contempla avaliação clínica, diagnóstico precoce, planejamento do tratamento, implementação do plano de cuidados e evolução das condutas e tratamento. Não se pode deixar 
em segundo plano a educação permanente da equipe, do próprio indivíduo e de sua família, quando se busca a organização assistencial (VASCONCELOS; CALIRI, 2017; ADAMY et al., 2018).

Entretanto, apenas um dos participantes identificou os protocolos assistenciais com uma importante ferramenta para tomada de decisões. Cita, por exemplo, os Procedimentos Sistêmicos (PRS):

"Temos alguns PRS sobre o manejo desses pacientes, porque se abre uma lesão, o risco para eles é grande e com isso vai impactar de uma forma geral. Então, esse cuidado tem que ser feito pela equipe multiprofissional: médico, enfermeiro, técnico de Enfermagem" (ENF 1).

O cuidado de Enfermagem sem suporte teórico e padronização adequados favorece 0 exercício profissional imperito, negligente ou imprudente, o que pode ocasionar danos ao usuário, problemas legais e éticos aos profissionais e descrédito da classe pela sociedade (PIMENTA et al., 2017). A sistematização dos processos de trabalhos facilita, auxilia e organiza a tomada de decisões, além de proporcionar o envolvimento da família no processo.

Os resultados evidenciaram a importância do acompanhamento diário para otimizar o plano de cuidados:

"Não temos muita ênfase em curativo e em cobertura. Chamamos o enfermeiro da comissão do curativo, para o controle eficaz da lesão, que é o objetivo da comissão. Temos que estar muito atentos em relação à friç̧ão, manter sempre o forro bem estendido, para não friccionar a pele do paciente" (ENF 8). "Nós instituímos as medidas preventivas. Podemos usar espumas com silicone, filme transparente para evitar uma infecção por lesão ou cisalhamento, coxins, as descompressões, a indicação de colchão pneumático, ajuda bastante a evitar esse tipo de lesão, além da nossa avaliação periódica" (ENF 6).

Ao conhecer e avaliar parte dos fatores de risco, as ações desenvolvidas individualmente ou em equipe permitem alcançar outras dimensões ou instrumentos/ferramentas para a atuação. Mas, os depoentes não deixaram de relatar algumas dificuldades em seu cotidiano.

\section{O olhar mais amplo para além das dificuldades na prevenção de lesões por pressão}

As necessidades do olhar de toda a equipe, além do apoio da família e de treinamentos permanentes, foram percebidas e verbalizadas como essencial para prevenir lesões na pele, decorrentes de pressão:

"A aplicação dessas ações e ferramentas é eficaz aqui. Educamos os familiares, inseridos nesse cuidado $e$ 
orientamos a equipe de Enfermagem. Quando se faz tudo da forma correta, quando se tem condições de fazer o que é certo, que é tirar a pressão, que é a única coisa que resolve para prevenir lesão e, quando a equipe tem condições de seguir os cuidados prescritos e educar os familiares, resolve esse problema, com certeza" (ENF 13).

"Os enfermeiros identificam e classificam os pacientes durante a internação em relação ao risco de lesão, prescrevem e os técnicos de Enfermagem, quando executam com eficiência e eficácia, o resultado é positivo" (ENF 11), "São vários olhares para o indivíduo: enfermeiro da unidade de internação, o da integridade cutânea, além dos técnicos de Enfermagem que dão banho no paciente todos os dias. Acredito que agimos antes de abrir a lesão" (ENF 7).

Mesmo reconhecendo todos os cuidados relevantes a serem prestados, os participantes relatam a dificuldade de realizar a assistência adequada, devido à escassez de recursos humanos, tanto de técnicos de Enfermagem, quanto de enfermeiros. Igualmente, sabe-se que a educação, como prática de cuidado, é essencial para a promoção da saúde, mas, os profissionais alegaram sobrecarga de trabalho e ausência de treinamentos específicos:

\begin{abstract}
"Não tem profissional suficiente para realizar a mudança de decúbito a cada duas horas. Não é tão efetivo como deveria ser, é um pouco falho nesse sentido, mas se a lesão existir e for aplicado o cuidado necessário, como tratar a lesão e prevenir quando ela não existe, então, realmente, seria o cuidado eficaz" (ENF 12). "Por causa da sobrecarga, boa parte dos colaboradores não contribui para isso. Por exemplo, mudança de decúbito não tem, acontece na hora das trocas de fralda e na hora do banho, mas aquela mudança de decúbito rigorosa, não acontece" (ENF 9). "Nosso problema é posterior: a reavaliação. Não é todo dia que eu consigo ir ao leito desse paciente para reavaliá-lo e evoluir, reaplicar 0 Braden, verificar se prescrição que eu fiz está sendo cumprida. É um processo que se perde e não depende somente do enfermeiro ou da equipe: demanda pessoal, conhecimento da parte técnica, treinamento" (ENF 2). "Se o paciente está com a mobilidade prejudicada, como as demandas são muitas, não consigo, estar presente ali, cobrando para poder observar. Então eu direciono o plano de cuidados, intensifico a questão da equipe para fazer a movimentação desse paciente, aviso o acompanhante para tal cobrança necessária, pois sei que, às vezes, não vai ser feito corretamente..." (ENF 3).
\end{abstract}

Ponto não menos importante refere-se ao trabalho em equipe, oportunidade em que se destaca o planejamento conjunto e as diferentes 
habilidades que se complementam com vistas à finalidade comum. Entretanto, mesmo diante da relevância do trabalho conjunto para um olhar mais amplo na prevenção das feridas, ainda existem deficiências no cuidado, devido à inadequação do quantitativo de recursos humanos necessários. Pesquisa realizada em duas instituições hospitalares destacou que na equipe de Enfermagem, a associação entre carga de trabalho e eventos adversos é significativamente alta. Favorece-se, assim, a incidência de tais ocorrências e os impactos negativos para a SP (COSTA et al., 2018).

Outro estudo observacional realizado em uma Unidade de Terapia Intensiva de Trauma acompanhou 195 pacientes. Constatou que os pacientes ali hospitalizados estariam suscetíveis a incidentes em relação ao tempo de internação, aos procedimentos, às intervenções terapêuticas e à carga de trabalho de Enfermagem. Nesse sentido, podem ser observados níveis elevados de estresse, ambiente de trabalho inadequado e insatisfação profissional, todos considerados fatores que comprometem a assistência segura (PADILHA et al., 2017). O que se almeja é a locação eficiente de recursos humanos para a consecução de resultados favoráveis e com menor risco assistencial, a partir da capacitação dos profissionais (FAGERSTROM; KINNUNEN; SAARELA, 2017; PADILHA et al., 2017).

Talvez por isso, os enfermeiros perceberam a necessidade de inserir 0 acompanhante/familiar no cuidado:

\begin{abstract}
"Verifico se é um paciente capaz de realizar a mudança de decúbito sozinho ou se vai depender de terceiros, para auxiliar, nesse cuidado, então, muitas vezes, é preciso inserir a equipe de Enfermagem nos cuidados com familiares que estão acompanhando" (ENF 5). "Não adianta você tratar um paciente, tratar uma lesão se o seu estado nutricional não está adequado, as doenças crônicas também são importantes nesse processo para intervenção. Além da mudança de decúbito, ela é essencial, e nem sempre tenho mão de obra disponível, por isso, sempre oriento o familiar a observar e acionar a Enfermagem, pois é importante dar continuidade ao cuidado" (ENF 1).
\end{abstract}

O envolvimento familiar surge como novo paradigma no contexto da atenção à saúde, não apenas para execução pura e simples de procedimentos pela escassez de profissionais nos diversos serviços de saúde. Promover a inserção e participação da família nesse cuidado é imprescindível pois, quando compreende e se torna corresponsável na prevenção, parte do protagonismo emerge no processo de viver saudável e no acompanhamento diário do indivíduo (BRASIL, 2017b; SOARES; HEIMANN, 2018).

Alguns participantes reconheceram prestar a melhor assistência possível, mesmo com algumas dificuldades, e que tudo tem início na avaliação eficiente, por ocasião da admissão hospitalar pois, em sua visão, seria o ponto de partida para sistematizar a assistência e minimizar os riscos para LP: 


\begin{abstract}
"Realmente quando avaliamos e existe o risco de lesão, a mudança de decúbito é feita e mais atenção em relação aos lençóis, secos, sem dobradura. Também ficamos atentos ao aporte nutricional e hidratação. Assim, se tudo é feito, como deve ser feito, não tem lesão" (ENF 10). "Quando temos uma boa avaliação do paciente conseguimos perceber risco para desenvolver lesão. Daí a importância do envolvimento de toda a equipe multiprofissional, para que a lesão não se instale. $A$ própria equipe de Enfermagem tem um papel fundamental, pois está com o paciente 24 horas por dia. $A$ sua assistência pode influenciar, ou não, na abertura da lesão" (ENF 6).
\end{abstract}

Isso posto, as LP são eventos evitáveis e relacionam-se intimamente com a SP, somados aos impactos financeiros, sociais e psicológicos para o indivíduo e para sua família (COSTA et al., 2018). Mas, é imprescindível conhecer para que a atuação profissional seja efetiva. Um estudo realizado com 40 profissionais de Enfermagem de um hospital universitário objetivou descrever e analisar o conhecimento dos profissionais acerca da classificação, avaliação e medidas de prevenção de LP. Os resultados apontaram conhecimentos insuficientes e necessidade de investimentos em capacitação (GALVÃO et al., 2017).

Conhecimento e sistematização assistencial contribuem para a qualidade do cuidado e devem ser o eixo na capacitação das equipes. Há que se avaliar se existe uma dicotomia entre o que se aprendeu, o que se faz, em quais condições e, principalmente, em relação ao que se desejaria ou deveria ser feito e que foi verbalizado pelos participantes.

O estudo possui a limitação de ter sido realizado em apenas uma instituição assistencial, mas, longe de generalizações, propõe a utilização dos achados nas reflexões e discussões em ambientes assistenciais para a qualificação do cuidar em saúde.

\title{
CONCLUSÃO
}

Os resultados fizeram emergir que apesar dos conhecimentos dos enfermeiros sobre os fatores de riscos intrínsecos e extrínsecos para o desenvolvimento de LP, as ferramentas disponíveis e a participação da equipe multiprofissional seriam suficientes para prevenir tais lesões. O papel das comissões especializadas é visto como definidor de intervenções eficazes, a partir da avaliação individual. Não deixam de ser passos ou etapas da SAE, reconhecidos, porém não inteiramente realizados, pois há a consideração das evidências favoráveis da identificação de riscos e da implementação de medidas preventivas. A família foi referendada como ator coadjuvante e a educação permanente qualifica o cuidado ao minimizar riscos para desenvolvimento de lesões relacionadas à pressão, durante a hospitalização. Há que se buscar o olhar mais apurado de gestores sobre os processos de 
trabalho de sua equipe, ao se considerar o potencial de preventabilidade das lesões pelo reconhecimento precoce dos fatores de risco.

\section{REFERÊNCIAS}

ADAMY, E. K. et al. Reflection on the interface between patient safety and the nursing process. Revista de Enfermagem e Atenção à Saúde, v. 7, n. 1, p. 272-278, 2018.

AGYEMAN-YEBOAH, J.; KORSAH, K. A.; OKRAH, J. Factors that influence the clinical utilization of the nursing process at a hospital in Accra, Ghana. BioMed Central Nursing, v.16, n.30, p. 1-7, 2017.

BARDIN, L. Análise de conteúdo. São Paulo: Edições 70, 2018, 280 p.

BRASIL. Ministério da Saúde. Agência Nacional de Vigilância Sanitária. Documento de referência para o Programa Nacional de Segurança do Paciente. Brasília: Ministério da Saúde, 2014. Disponível em: http://bvsms.saude.gov.br/bvs/publicacoes/documento_referencia_programa_n acionalseguranca.pdf. Acesso em: 16 set. 2018.

BRASIL. Ministério da Saúde. Agência Nacional de Vigilância Sanitária. Boletim Segurança do Paciente e Qualidade em Serviços de Saúde no 15: Incidentes Relacionados à Assistência à Saúde. Brasília: Ministério da Saúde, 2017a.

BRASIL. Ministério da Saúde. Agência Nacional de Vigilância Sanitária. Nota Técnica GVIMS/GGTES № 03/2017. Práticas seguras para prevenção de Lesão por Pressão em serviços de saúde, Brasília: Ministério da Saúde, 2017b. Disponível em: http://portal.anvisa.gov.br/documents/33852/271855/Not $\mathrm{a}+\mathrm{T} \% \mathrm{C3} \%$ A9cnica+GVIMS-GGTES+n\%C2\%BA+03-2017/54ec39f6-84e0-4cdb -a241-31491ac6e03e?version=1.0. Acesso em: 12 dez. 2018.

CAMARA, R. H. Content analysis: from theory to practice in social research applied to organizations. Gerais: Revista Interinstitucional de Psicologia, v. 6, n. 2, p. 179-191, 2013. Disponível em: http://pepsic.bvsalud.org/pdf/gerais/ v6n2/v6n2a03.pdf. Acesso em: 4 set. 2018.

COSTA, D. B. et al. Patient safety culture: evaluation by nursing professionals. Texto \& Contexto - Enfermagem, v. 27, n. 3, 2018.

COX, J.; ROCHE, S.; MURPHY, V. Pressure injury risk factors in critical care patients: a descriptive analysis. Advances in Skin \& Wound Care, v. 31, n. 7, p. 328-334, 2018.

FAGERSTROM, L.; KINNUNEN, M.; SAARELA, J. Nursing workload, patient safety incidents and mortality: an observational study from Finland. BioMed Journal Open, v. 8, 2018. 
GALVÃO, N. S. et al. Knowledge of the nursing team on pressure ulcer prevention. Revista Brasileira de Enfermagem, Brasília, v. 70, n. 2, p. 294300, 2017.

GOMES, A. et al. Patient safety in nursing paths in Brazil. Revista Brasileira de Enfermagem, Brasília, v. 70, n. 1, p. 146-154, 2017.

HOMMEL, A.; SANTY-TOMLINSON, J. Pressure injury prevention and wound management. In: Hertz K., Santy-Tomlinson J. (eds). Fragility Fracture Nursing. Perspectives in Nursing Management and Care for Older Adults. Switzerland: Springer, 2018.

MACHADO, F. S. et al. Perspective of the nurse in front of nursing care in the treatment of wounds in hospitals. Journal of Epidemiology and Infection Control, Santa Cruz do Sul, [S.I.], v. 7, n. 3, p. 134-139, 2017.

MASCARENHAS, S. N.; MELO, C. M. M. Erros assistenciais e o processo de trabalho em enfermagem no hospital. 2015. 106 f. Dissertação (Mestrado em Enfermagem) - Escola de Enfermagem, Universidade Federal da Bahia, Salvador, 2015. Disponível em: https://repositorio.ufba.br/ri/handle/ri/20106. Acesso em: 10 nov. 2018.

MORAES, J. T. et al. Conceito e classificação de lesão por pressão: Atualização do National Pressure Ulcer Advisory Panel. Revista de Enfermagem do Centro Oeste Mineiro, v. 6, n. 2, p. 2292-2306, 2016.

PADILHA, K. G. et al. Nursing workload, stress/burnout, satisfaction and incidents in a trauma intensive care units. Texto \& Contexto - Enfermagem, v. 26, n. 3, e1720016, 2017.

PIMENTA, C. A. M. et al. Guia para Construção de Protocolos Assistenciais de Enfermagem. São Paulo: COREN-SP, 2017. Disponível em: https://portal.coren-sp.gov.br/sites/default/files/Protocolo-web.pdf. Acesso em: 16 set. 2018.

PRODANOV, C.; FREITAS, E. Metodologia do trabalho científico: métodos e técnicas da pesquisa e do trabalho acadêmico. 2 ed. Novo Hamburgo: Feevale, 2013. Disponível em: http://www.feevale.br/Comum/midias/8807f05a14d0-4d5b-b1ad-1538f3aef538/E-book\%20Metodologia\%20do\%20Trabalho\%2 0Cientifico.pdf. Acesso em: 11 ago. 2018

ROCHA, S. C. G. et al. Comparison of pressure injury risk assessment scales. Revista Brasileira de Pesquisa em Saúde, v. 18, n. 4, p. 143-151, 2016.

SALGADO, L. P. et al. Escalas preditivas utilizadas por enfermeiros na prevenção de lesão por pressão. Saber Digital, v. 11, n. 1, p. 18-35, 2018. Disponível em: http://revistas.faa.edu.br/index.php/SaberDigital/article/vie 
w/468. Acesso em: 10 jan. 2019.

SILVA, A. et al. Patient safety in the hospital context: an integrative literature review. Revista Cogitare Enfermagem, v. 21, n. esp: 01-09, 2016.

SOARES, C. F.; HEIMANN, I. T. S. Health promotion and prevention of pressure injury: expectations of primary health care nurses. Texto \& Contexto Enfermagem, Florianópolis, v. 27, n. 2, 2018.

VASCONCELOS, J.; CALIRI, M. Nursing actions before and after a protocol for preventing pressure injury in intensive care. Escola Anna Nery, v. 21, n. 1, 2017.

ZIMMERMANN, G. S. et al. Pressure injury risk prediction in critical care patients: an integrative review. Texto \& Contexto - Enfermagem, Florianópolis, v. 27, n. 3, 2018. 